\title{
Normality of single fibre electromyographic jitter: a new approach
}

\author{
D J BAKER, N L CROSS, E M SEDGWICK*
}

From the Chemical Defence Establishment, Porton Down, Salisbury, and the ${ }^{*}$ Wessex Neurological Centre, Southampton General Hospital, Southampton, UK

SUMMARY The distribution of jitter values derived from single fibre electromyography (SFEMG) in normal extensor digitorum communis has been found to be skewed. A reciprocal transformation technique is presented which renders the distribution normal. The distribution enables SFEMG changes to be assessed using parametric statistics. The relationship of the new technique to existing methods of analysis of SFEMG is discussed.

The technique of single fibre electromyography (SFEMG) ${ }^{12}$ has become an established method of detecting abnormalities of neuromuscular transmission in many conditions affecting the end plate and shows considerable potential for pharmacological studies of drugs affecting cholinergic transmission. ${ }^{3}$ Conventional clinical SFEMG practice involves recording up to 20 muscle fibre pairs from one or more muscles and examining the jitter values so obtained against comparable control data. Several authors have published data for normal muscles, notably for extensor digitorum communis. The upper limit of normal jitter is usually taken as being three standard deviations above the unweighted mean for the whole population of jitters. For extensor digitorum communis, the accepted upper limit of the mean consecutive difference (MCD) of normal jitter has been established as $55 \mu \mathrm{s} .{ }^{45}$ These studies have shown that in a large series of recordings only one fibre pair in twenty is above this value. The diagnostic yield of clinical SFEMG examination has been increased by using the mean value of jitter from the recorded fibre pairs and comparing them with values from control populations. Using this approach the value of $34 \mu \mathrm{s}$ has been established by Stalberg ${ }^{4}$ and Saunders ${ }^{5}$ as the upper limit of normal mean jitter. The mean in the latter case was calculated from a distribution of jitter values truncated above $55 \mu \mathrm{s}$.

Address for reprint requests: Dr EM Sedgwick, Wessex Neurological Centre, Southampton General Hospital, Southampton SO9 4XY, UK.

Received 15 April 1986. Accepted 19 June 1986
One problem arising in the use of SFEMG as a pharmacological tool is the presence of skew in the distribution of recorded jitter values. In dealing with any possible drug action at the neuromuscular end plate an analytical technique is required which can compare effects upon the whole jitter population and which can deal with possible dose related changes in the degree of skew. This is important given the morphological and possibly physiological differences shown by end plates. ${ }^{6}$ Simple comparison of unweighted means of jitter values using parametric statistical methods is not possible while the skew error remains in the distribution.

This paper describes a technique of reciprocal transformation of jitter values to produce a near normal distribution. The transformation allows SFEMG jitter changes in a whole population of fibre pairs to be compared using the Student $t$, or any other parametric test.

\section{Methods}

SFEMG recordings were made in the extensor digitorum communis muscle of $25 \mathrm{fit}$, male volunteers at the Chemical Defence Establishment, Porton Down. The data were part of a double blind study of the effects of pyridostigmine and low dose organophosphate on SFEMG. ${ }^{7}$ Each volunteer was fully briefed about the experimental protocol. The studies were conducted in accordance with the WHO international guidelines for human studies $(1982)^{8}$ and were controlled by internal and external ethical committees.

SFEMG was recorded from the right forearm during voluntary activation of the extensor digitorum communis by extension of the third finger. The recording technique was identical to that described by Eckstedt and Stalberg. ${ }^{1}$ Signals 
from the electrode (S37, Medelec, England) were amplified and processed using an MS6 electromyograph (Medelec) and recorded at 15 inches/second on a reel to reel FM tape recorder (SE 84, England) for off-line calculation of jitter. MCD values were derived using an Apple IIe microcomputer linked with the electromyograph through a CI6 interface unit. Each MCD value was computed from 40 consecutive discharges at an activation frequency of between 15 and $20 \mathrm{~Hz}$. Recordings were made in quiet conditions with the subject relaxed, lying on a bed. These conditions were found to be important in view of the recognised difficulty in recording technically acceptable SFEMG from fit, young subjects.

\section{Statistical methods}

The fit of data to a normal distribution was assessed using standard calculations of skewness and kurtosis. ${ }^{9}$ In addition the Kolmogorov D statistic, ${ }^{10}$ which is a sensitive test of normal distribution was used. Data were processed using a Tektronix 4051 (Tektronix UK Ltd) computer with a standard statistical software library.

\section{Results and analysis}

During the study, 421 technically acceptable fibre pairs were recorded during control SFEMG recording sessions in 25 subjects. These data have been pooled to give the distribution of MCD values shown in fig 1 . The overall mean MCD is $30 \cdot 11 \pm 29.97$ (SD) $\mu$ s and the range of values was $10-315 \mu \mathrm{s}$. Twenty fibre pairs $(4.7 \%)$ in the original data have MCD values greater than $55 \mu \mathrm{s}$. Figure 2 shows the data from fig 1 replotted with values of MCD $>55 \mu$ s truncated. The mean MCD value of this distribution is now $25 \cdot 37 \pm$ $9 \cdot 29 \mu \mathrm{s}$. To examine the closeness of these distributions to the normal, data from figs 1 and 2 are plotted as conventional cumulative frequency plots in figs $3 \mathrm{a}$ and $b$. In this method a normal distribution appears as a straight line. The marked deviation from linearity in both non-truncated and truncated data indicates a

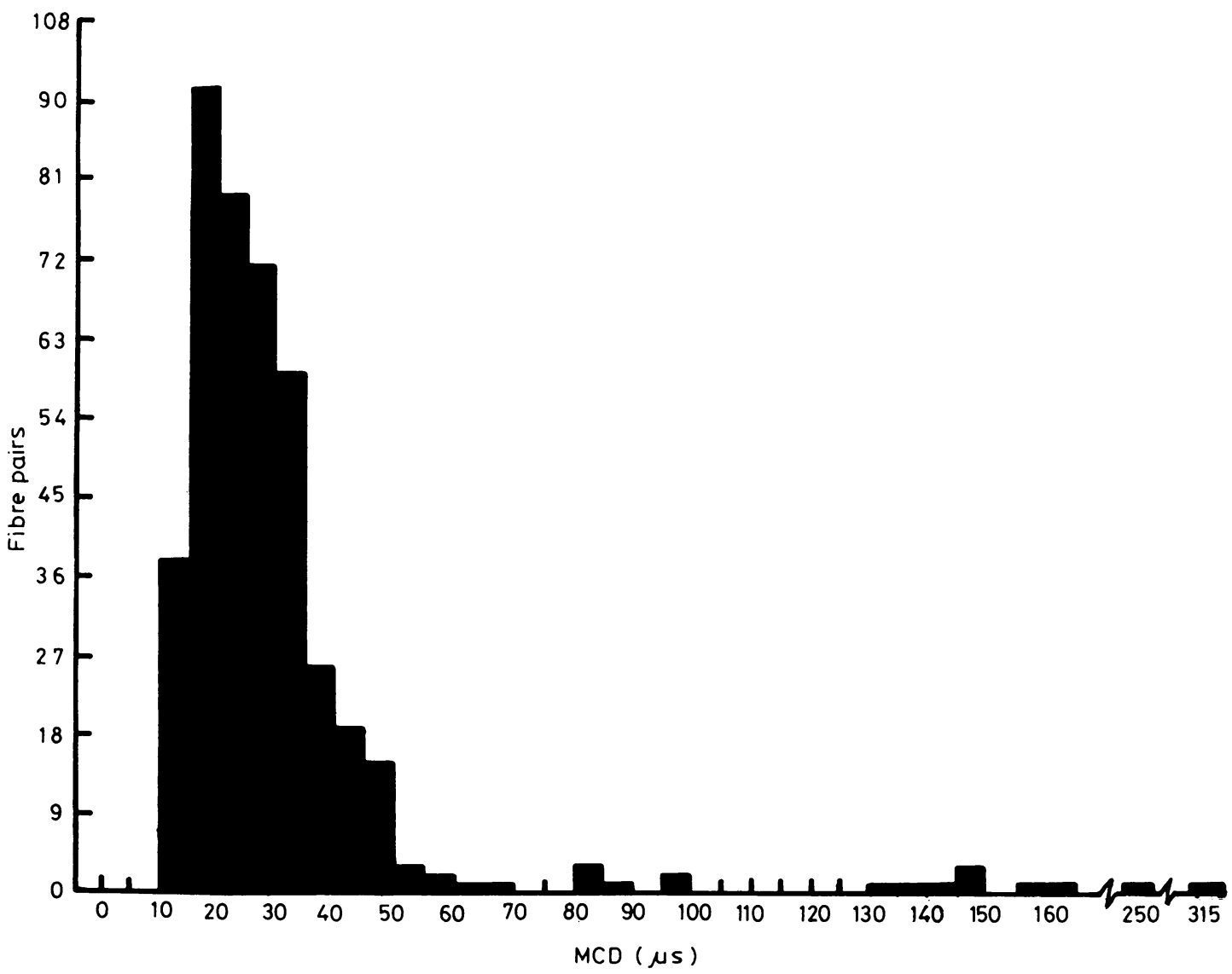

Fig 1 Distribution of SFEMG jitter from 421 fibre pairs recorded in extensor digitorum communis of 25 normal males. Jitter values are expressed as mean consecutive difference (MCD) in $\mu s$. 


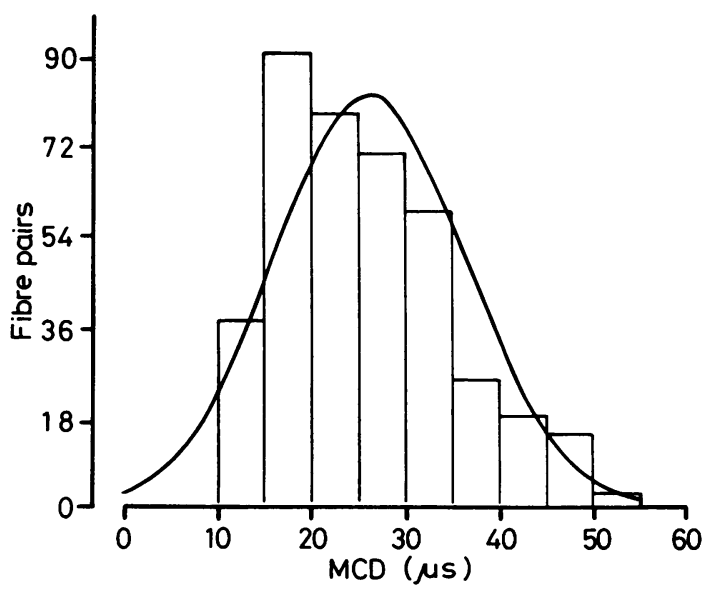

Fig 2 Data from fig 1 redrawn with $M C D$ values $>55 \mu$ s and $<10 \mu s$ truncated. The distribution appears skewed in comparison with the normal distribution shown superimposed as a solid line.

departure from the normal distribution in both. This departure is confirmed by examination of the skewness, kurtosis and Kolmogorov D statistics shown in the table.

\section{Reciprocal transformation}

The standard practice in dealing with data which do not fit a normal distribution is to subject them to a mathematical transformation and then examine the normality of the distribution of the modified values. The most usual transformation with biological data is logarithmic. This approach was tried but produced no improvement in the data presented here. Reciprocal transformation however was shown to produce considerable improvement. In this process MCD values were transformed according to the relationship:

$$
\mathrm{MCD}^{-1}=1000 / \mathrm{MCD}
$$

The reciprocal transform for each jitter value has the dimension of $1 / T$ and may conveniently be expressed in $\mathrm{kHz}$ when the MCD is in $\mu \mathrm{s}$. The reciprocal MCD parameter has been termed "jitter frequency".

Figure 4 shows the distribution of data from fig 1 after reciprocal transformation. The mean value of jitter frequency is $43.3 \pm 17.9 \mathrm{kHz}(421)$ and the range of values is $3 \cdot 2-100 \mathrm{kHz}$. The cumulative frequency plot of the transformed data, shown in fig $3 \mathrm{c}$, now displays little departure from linearity. Inspection of the statistics for the transformation shows that transformation produces marked reduction in the degree of skewness displayed by the original data and the values of kurtosis and Kolmogorov $D$ are reduced to insignificance. In comparison with the statistics for the truncated distribution, reciprocal transformation
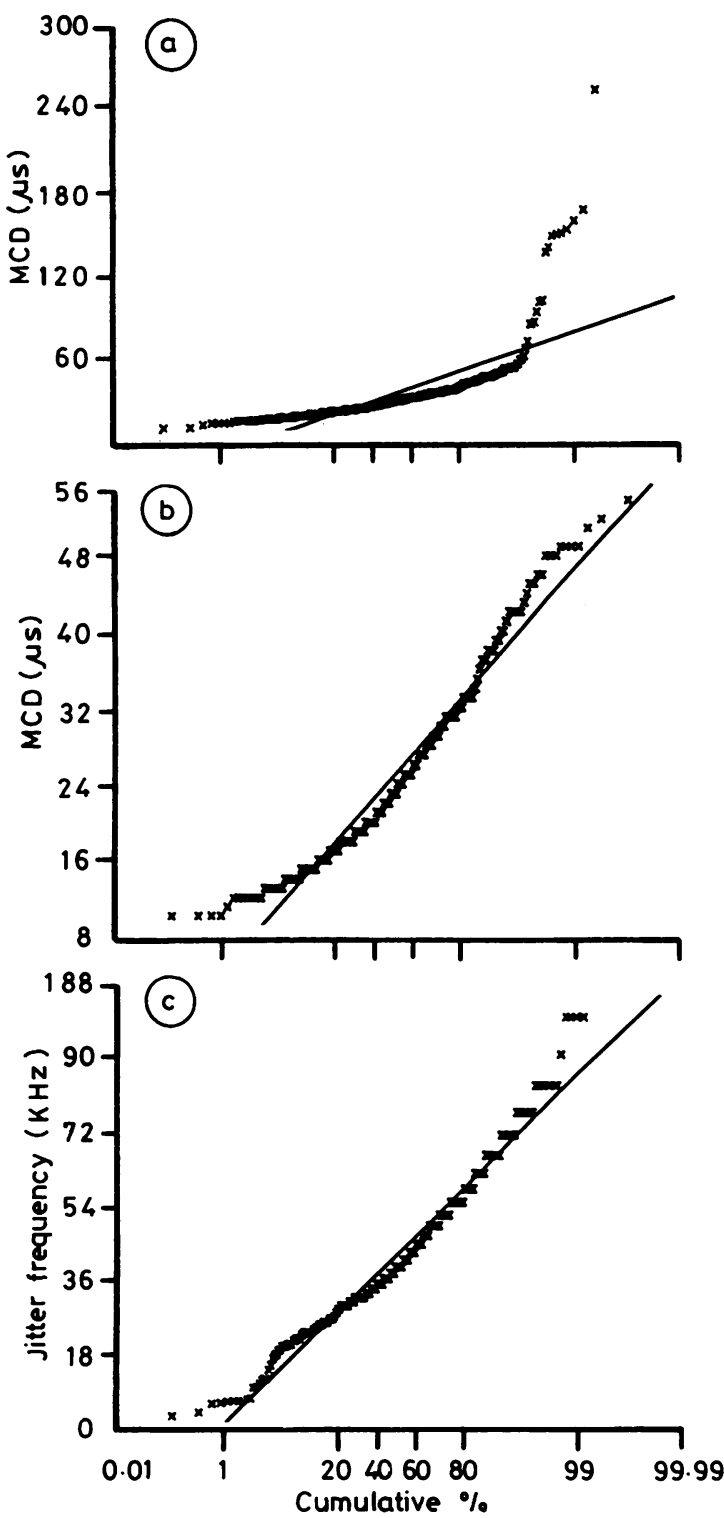

Fig 3 (a) Cumulative frequency plot of the distribution of $M C D$ values presented in fig 1 . The data do not follow a normal distribution. The non linearity of the data is evident in comparison with the superimposed straight line of a normal distribution. (b) Cumulative frequency plot of truncated data in fig 2. Although high jitter values have been eliminated there is still a departure from a normal distribution. (c) Cumulative frequency plot of reciprocally transformed data from fig 4 . There is closer approximation to a normal distribution than in fig $3 b$ although all technically acceptable data points have now been included. Comparison with fig $3 a$ shows the closeness to normal distribution produced by reciprocal transformation of the complete data set. 
Table SFEMG: distribution parameters of alternative transformations

\begin{tabular}{|c|c|c|c|}
\hline \multirow[b]{2}{*}{$\begin{array}{l}\text { Statistic } \\
n\end{array}$} & \multicolumn{2}{|c|}{ Untransformed data } & \multirow[b]{2}{*}{$\begin{array}{l}\text { Reciprocal transformation } \\
\text { All data } \\
421 \\
\text { Jitter frequency }(\mathrm{kHz})\end{array}$} \\
\hline & $\begin{array}{l}\text { All data } \\
421 \\
M C D(\mu s)\end{array}$ & $\begin{array}{l}\text { Truncated data } \\
401\end{array}$ & \\
\hline $\begin{array}{l}\text { Mean } \\
\text { SD } \\
\text { Range } \\
\text { Skewness } \\
\text { Kurtosis } \\
\text { Kolmogorov D }\end{array}$ & $\begin{array}{l}30.11 \\
29.97 \\
10-315 \\
5.78, p<0.001 \\
47.48, p<0.001 \\
0.228, p<0.01\end{array}$ & $\begin{array}{l}25.37 \\
9.29 \\
10-54 \\
0.69, p<0.001 \\
2.91, p=0.36 \\
0.096, p<0.01\end{array}$ & $\begin{array}{l}43 \cdot 31 \\
17 \cdot 88 \\
3 \cdot 2-100 \\
0.50, \mathrm{p}<0.001 \\
3 \cdot 23, \mathrm{p}=0.17 \\
0.08,0.01<\mathrm{p}<0.05\end{array}$ \\
\hline
\end{tabular}

is seen to produce a closer fit to a normal distribution while including all the technically acceptable data.

\section{Discussion}

The clinical diagnostic use of SFEMG involves recording up to 20 muscle fibre pairs and examining the spread of the jitter values obtained. The basis for this method is the observation from large series of control recordings of a fixed incidence of MCD values greater than three standard deviations above the mean. In the case of extensor digitorum communis, the upper limit of normal jitter has been established as being $55 \mu \mathrm{s}$ and in normal subjects only $5 \%$ of recorded jitters may be expected to exceed this value in a large series. ${ }^{4}$ This criterion has proved useful in assessing muscle affected by pathological conditions such as myasthenia gravis, where the incidence of abnormal jitter is often very high. Diagnostic yield from SFEMG has also been improved by determining the mean jitter value. ${ }^{5}$

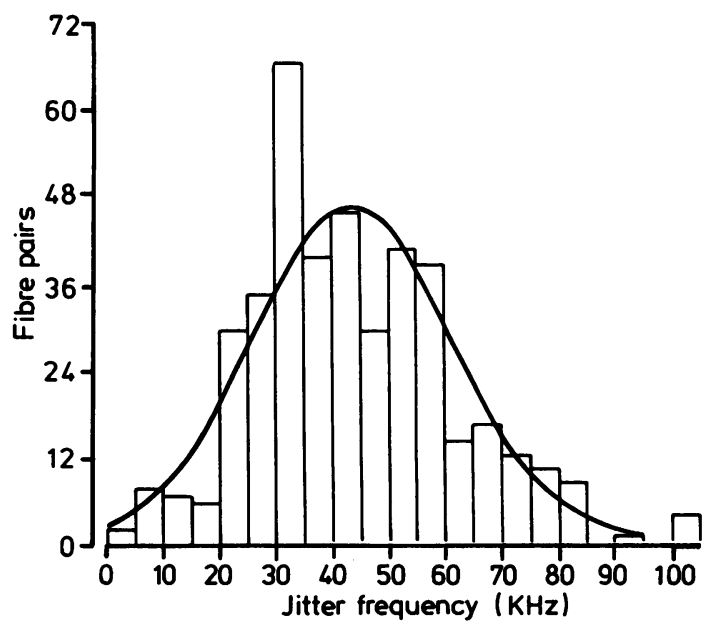

Fig 4 Reciprocal transformation of data from fig 1. Transformed $M C D$ values are shown as $f(k H z)$ corresponding to $1 / \mu s$.
However, in situations where only minimal changes in jitter may be expected certain problems arise in applying the standard analytical approach. The first is that, using the high jitter cut-off point, to be $95 \%$ certain that a muscle is normal (that is, that less than $5 \%$ of MCD values are greater than $55 \mu \mathrm{s}$ ) examination of tables of exact confidence ${ }^{11}$ shows that at least 72 fibre pairs must be recorded, none of whose MCD values must exceed $55 \mu \mathrm{s}$. Conversely, to be $95 \%$ certain that a muscle is abnormal according to the high jitter incidence rule, at least $3 / 10,5 / 30,8 / 70$ or $11 / 110$ responses must be greater than $55 \mu \mathrm{s}$.

The second problem with the conventional method of jitter analysis is the existence of skew in the distribution. Truncating the upper tail of such a distribution is the essence of the high jitter incidence rule. However, comparison of simple means from skewed distributions is not a satisfactory way of assessing shifts in a range of jitter values, since conventional parametric statistics such as the $t$ test cannot be used.

To determine the effect of any drug on neuromuscular transmission, the whole recorded range of jitter values should be used, since it is possible that not only the magnitude of individual jitter values but also the shape of their distribution may be affected by variations in safety factor within a population of muscle fibres. High jitter values, for example, have been shown to be more sensitive to the effects of non depolarising neuromuscular blocking drugs. ${ }^{3}$

Moreover, in human studies, where test doses of drugs are small, any observed effect may be minimal. The use of a non parametric statistical method such as the Wilcoxon Rank Sum test to determine such drug effects is limited by its relative insensitivity.

SFEMG recordings in 25 volunteers have shown that the value of mean control jitter from a distribution truncated at $55 \mu \mathrm{s}$ is $25.37 \pm 9.29 \mu \mathrm{s}$. This value is in good agreement with results published by other workers. However, the distribution of our data showed a marked deviation from the normal. Application of parametric testing to experimentally induced changes in the population mean is inappropriate in such a situation. The technique of reciprocal transformation of MCD values of jitter produces a distri- 
bution which is close to normal, as judged by the cumulative frequency plot and calculation of the skewness, kurtosis and Kolmogorov D statistics. The transformation is an improvement over the conventional method of truncating the distribution of MCD. Although the latter method produces a closer approximation to a normal distribution it does so only at the expense of rejecting data points with high jitter. Reciprocal transformation is suggested as being a sensitive way of examining the effects of drugs on jitter and has been used in a study of the effects of anticholinesterases on SFEMG. ${ }^{7}$

\section{References}

1 Eckstedt J, Stalberg E. Single fibre EMG for the study of the microphysiology of human muscle. In: Desmedt JE, ed. New Developments in Electromyography and Clinical Neurophysiology. Basel: Karger, 1973:157-61.

2 Stalberg E, Trontelj J. Single Fibre Electromyography. Old Woking, Surrey: Mirvalle Press, 1979.

3 Schiller HH, Stalberg E, Schwartz MS. Regional curare for the reduction of the safety factor in human motor end plates studied with single fibre electromyography. J Neurol Neurosurg Psychiatry 1975;38:805-9.

4 Stalberg E, Eckstedt J, Broman A. The electromyographic jitter in normal human muscles. Electroencephalogr Clin Neurophysiol 1971;31:429-38.

5 Saunders BD, Howard JF, Johns TR. Single fibre electromyography in myasthenia gravis. Neurology 1979;29:68-76.

6 Duchen LW, Gale AN. The motor end plate. In: Swash M, Kennard C, eds. Scientific Basis of Clinical Neurology. Edinburgh: Churchill Livingstone, 1985:400-9.

7 Baker DJ, Sedgwick EM. Anticholinesterases and single fibre electromyography. Electroencephalogr Clin Neurophysiol 1985;61:S85.

8 WHO Guidelines for Human Experiments. Geneva: CIOMS, 1982.

9 Snedecor CW, Cochran WG. Statistical Methods. 6th ed. Iowa State University Press, 1967:86.

10 Siegel S. Nonparametric Statistics for Behavioural Sciences. New York: McGraw Hill International Student Edition, 1956.

11 Lentner C. CibaGeigy Scientific Tables. 8th ed. Basle, 1982:89-107. 